\title{
Novel High Temperature Capacitive Pressure Sensor Utilizing SiC Integrated Circuit Twin Ring Oscillators
}

M. Scardelletti, P. Neudeck,

ECI@2 The 67th Electronic Components and Technology Conference
D. Spry, R. Meredith, J. Jordan, N. Prokop, M. Krasowski, G. Beheim and G. Hunter NASA Glenn Research Center

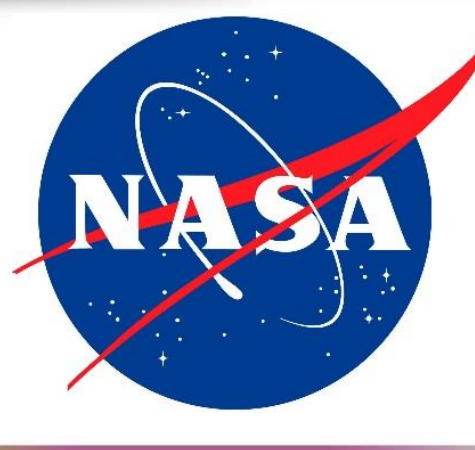

May 30 - June 2, 2017 


\section{Introduction}

- Exploration of planets and other celestial bodies is essential in order to determine the origins and future environmental conditions on Earth.

- Venus (our sister planet) especially noteworthy due similarities to Earth (size and make-up)

- But the two planets evolved very differently

$>$ Venus lacks oceans

$>$ Thick $\mathrm{CO}_{2}$ atmosphere

$>$ Surface temperature $470^{\circ} \mathrm{C}$ and pressure $1350 \mathrm{psi}$

$>$ Very caustic containing enough $\mathrm{SO}_{2}$ to form large sulfuric acid clouds tens kilometers thick in the upper atmosphere 


\section{Introduction}

However Venus may have had shallow liquid-water oceans and habitable surface temperatures for up to 2 billion years in its early history....... What happened??????

Fundamental questions about the nature of Venus remain shrouded due to:

- The huge challenge of returning useful scientific data from this hostile surface environment

- Only possible to return more than two hours of meteorological and seismic observational data from landers with current electronics and sensors 


\section{Introduction}

\section{Electronics and sensors designed for harsh environments}

Wide bandgap semiconductors
4-H SiC JFET integrated circuits

GEER (Glenn Extreme Environments Rig) Chamber

Three weeks of operation in Venus-like conditions

The Long-Life In-situ Solar System Explorer (LLISSE) project at NASA Glenn Research Center 


\section{Introduction}

The presentation will describe the initial development of a high temperature capacitive pressure sensor system for the surface of Venus

- SiC IC twin ring oscillators and SiCN capacitive pressure sensor

- Keysight's Advanced Design System circuit simulator

- 25 to $500^{\circ} \mathrm{C}$ and 0 to 300 psi operation

- Measured with and without sensor to demonstrate the effects of the sensor

- The sensor system illustrates the initial Earth-like condition testing as a step to long periods of time on the surface of Venus 


\section{1-Stage Ring Oscillator Design}

- NOT logic gate stage

$>\quad 2$ JFET (Models for 25 and $500^{\circ} \mathrm{C}$ )

$>\quad 3$ resistors (Models for 25 and $500^{\circ} \mathrm{C}$ )

$>1$ capacitor (parasitic effects)

- 11-satge ring oscillator

$>11$ NOT logic gates placed in a series feedback ring

$>$ Two Not gate buffers for output signal

- Capacitive pressure sensor

$>$ Pressure sensor is connected to one node of the feedback loop and to

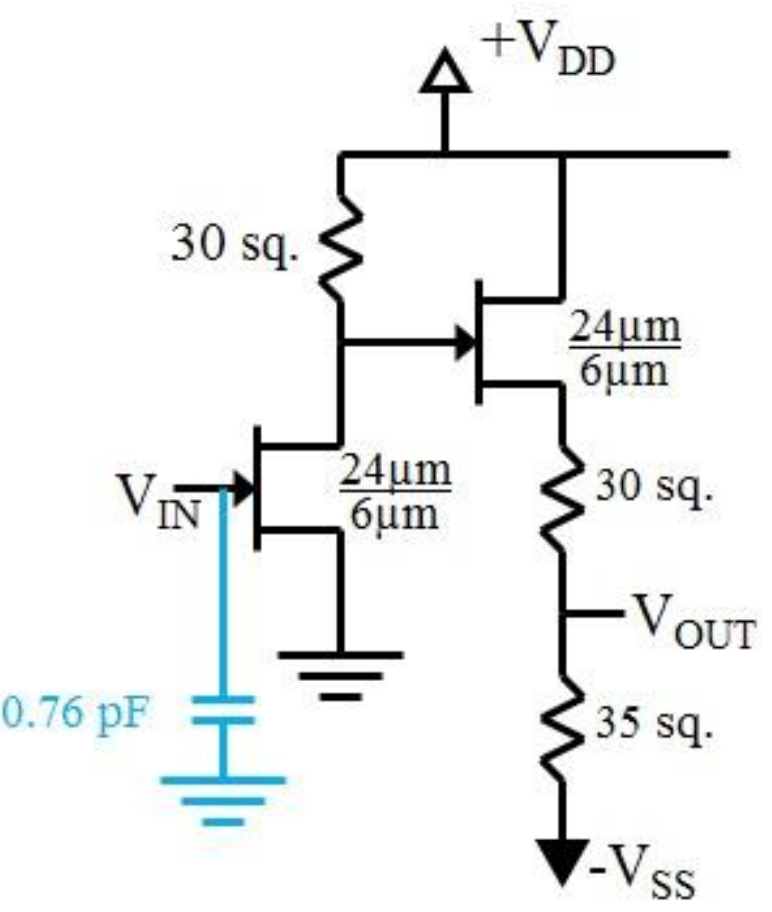

Single stage NOT Gate schematic ground (modeled as capacitor) 


\section{1-Stage Ring Oscillator Design}

11-stage ring oscillator with buffer gates and capacitor pressure sensor

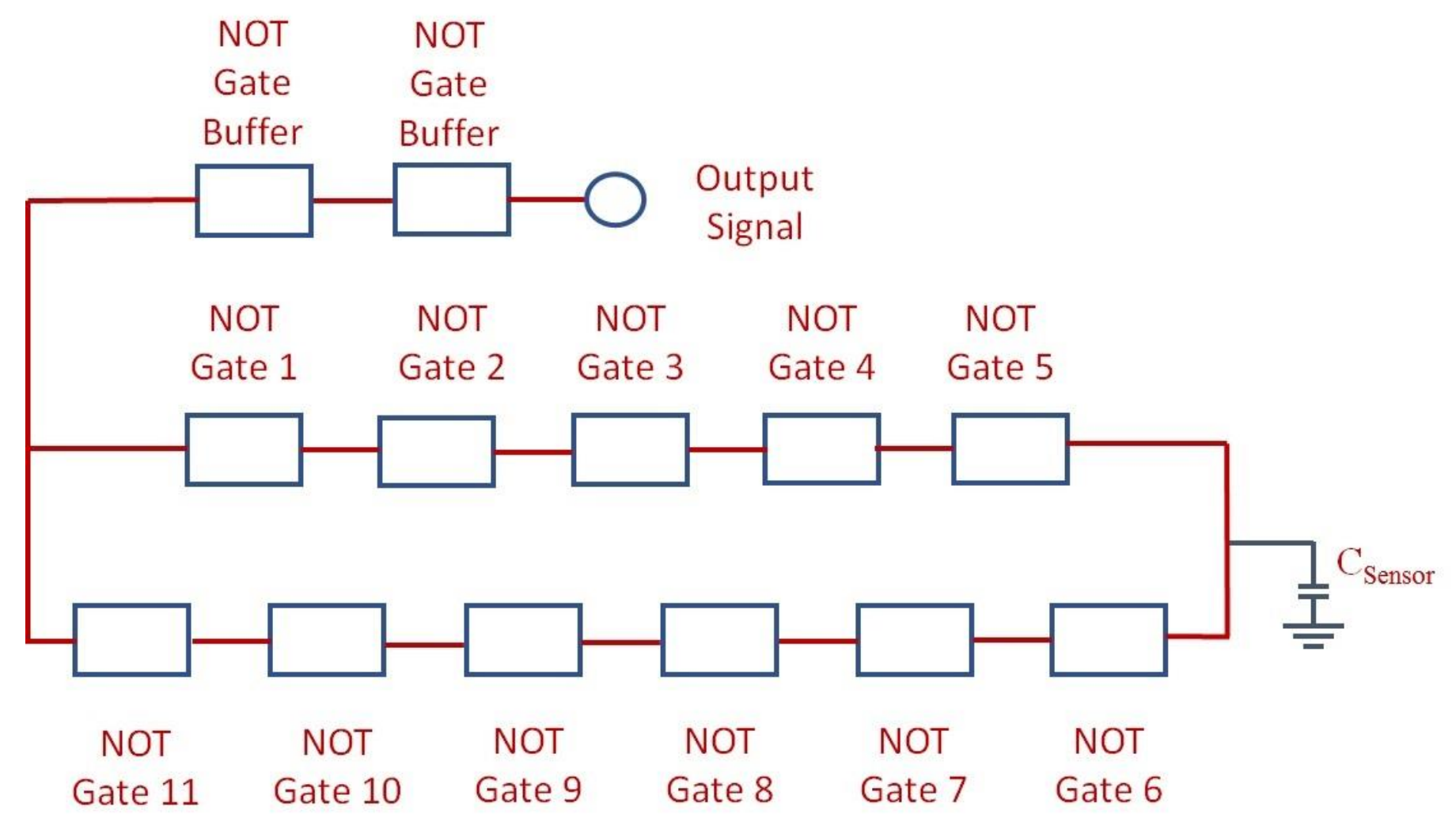




\section{Simulations}

Simulations performed in Keysight's Advanced Design System (ADS) Software Circuit Simulator

- JFET models parameters extracted from material and circuit evaluation

- Models for 25,300 and $500^{\circ} \mathrm{C}$ developed

- IV-curves simulations

- Harmonic Balance simulations

- Transient Analysis 


\section{Simulations}

\section{Harmonic Balance simulations of 11-stage ring oscillator with $\mathrm{NO}$ sensor at 25 and $500^{\circ} \mathrm{C}$}

$500^{\circ} \mathrm{C}$ simulated results $25^{\circ} \mathrm{C}$ simulated results
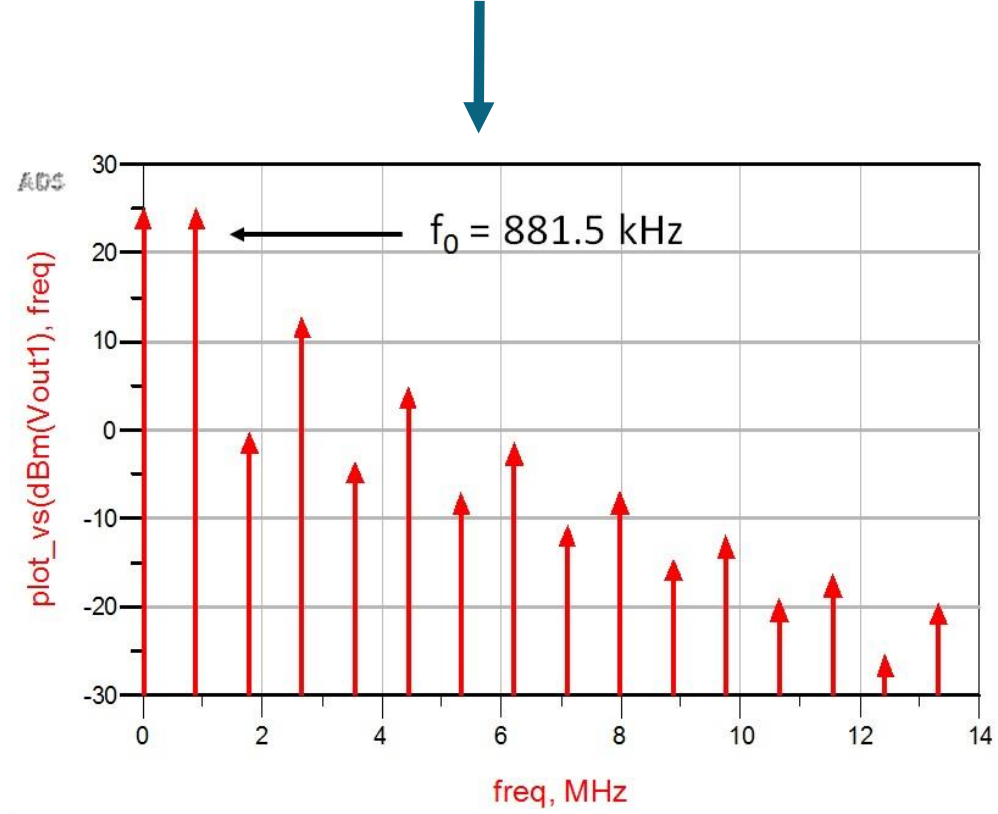

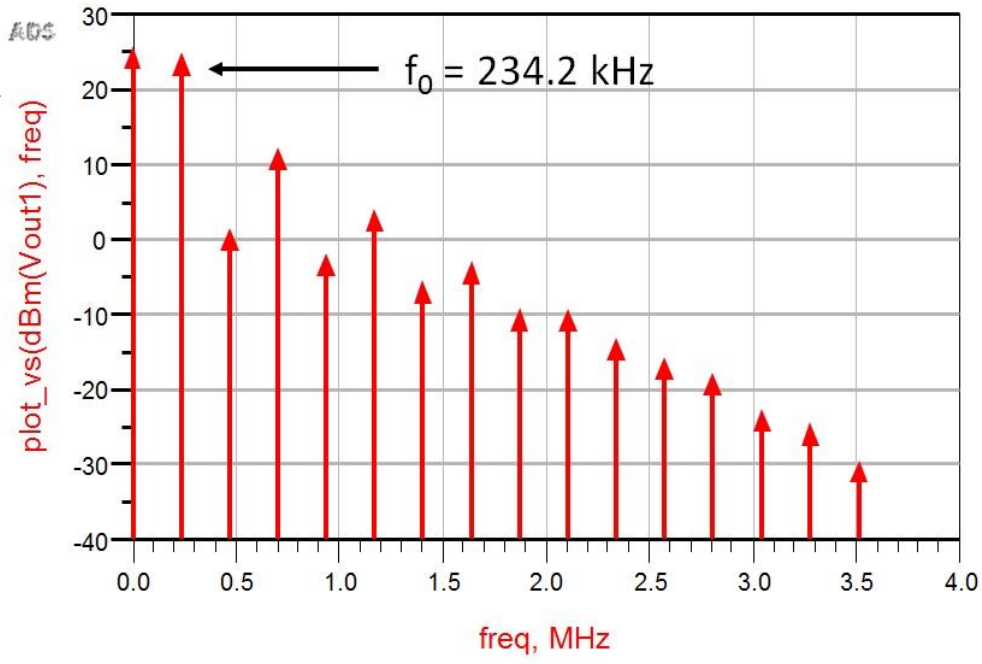

The resonate frequency decreases from $881.5 \mathrm{kHz}$ at $25^{\circ} \mathrm{C}$ to $234.2 \mathrm{kHz}$ at $500^{\circ} \mathrm{C}$, which is a reduction of 647.3 $\mathrm{kHz}$ or $73.4 \%$ from 25 to $500^{\circ} \mathrm{C}$ 


\section{Simulations}

\section{Transient Analyses simulations of 11-stage ring oscillator with $\mathrm{NO}$ sensor at 25 and $500^{\circ} \mathrm{C}$}

$500^{\circ} \mathrm{C}$ simulated results
$25^{\circ} \mathrm{C}$ simulated results
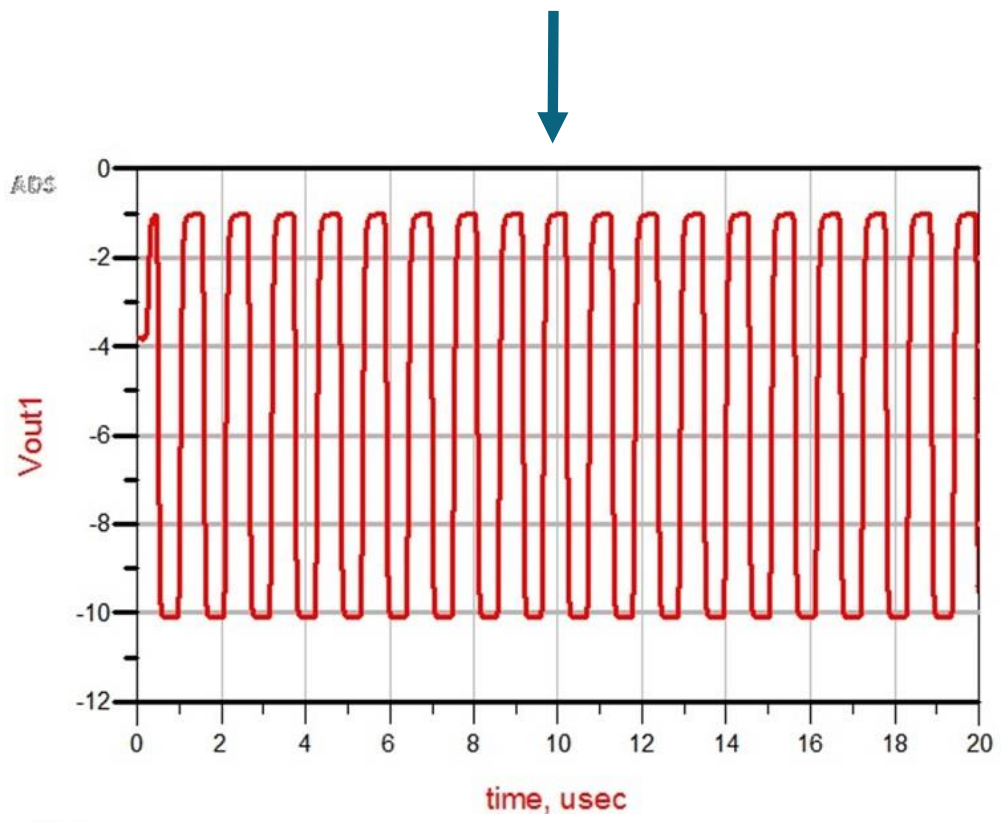

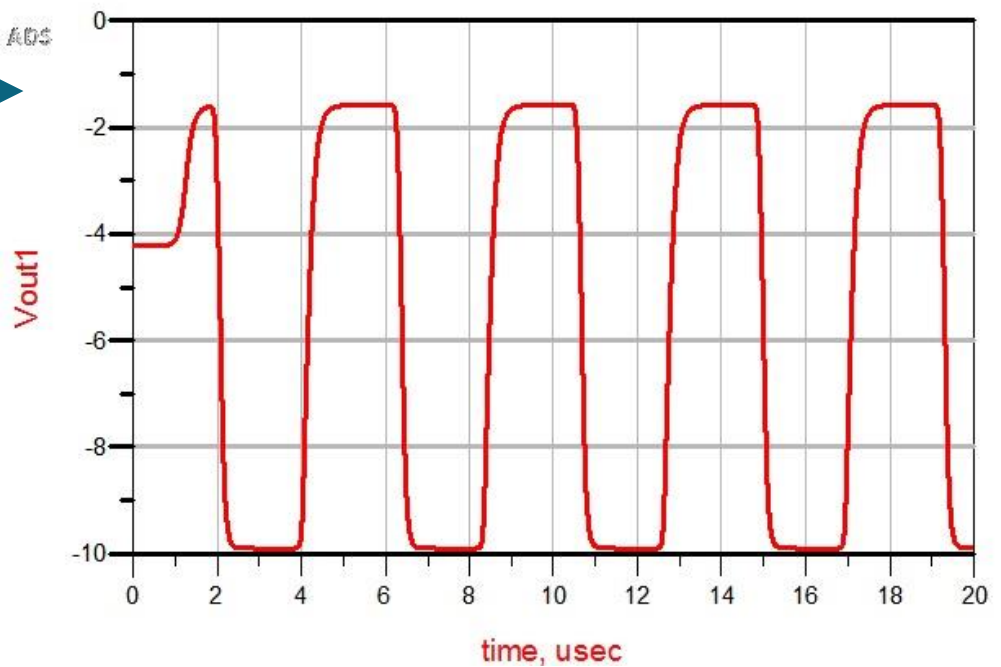

Transient simulated behavior illustrates same reduction in frequency from 25 to $500^{\circ} \mathrm{C}$ as expected 


\section{Fabricated 11-stage ring oscillator}

Fabricated 4-H SiC integrated circuit technology developed at NASA GRC exhibiting thousands of hours at $500^{\circ} \mathrm{C}$ in Earth-like conditions and over 500 hours Venus-like conditions

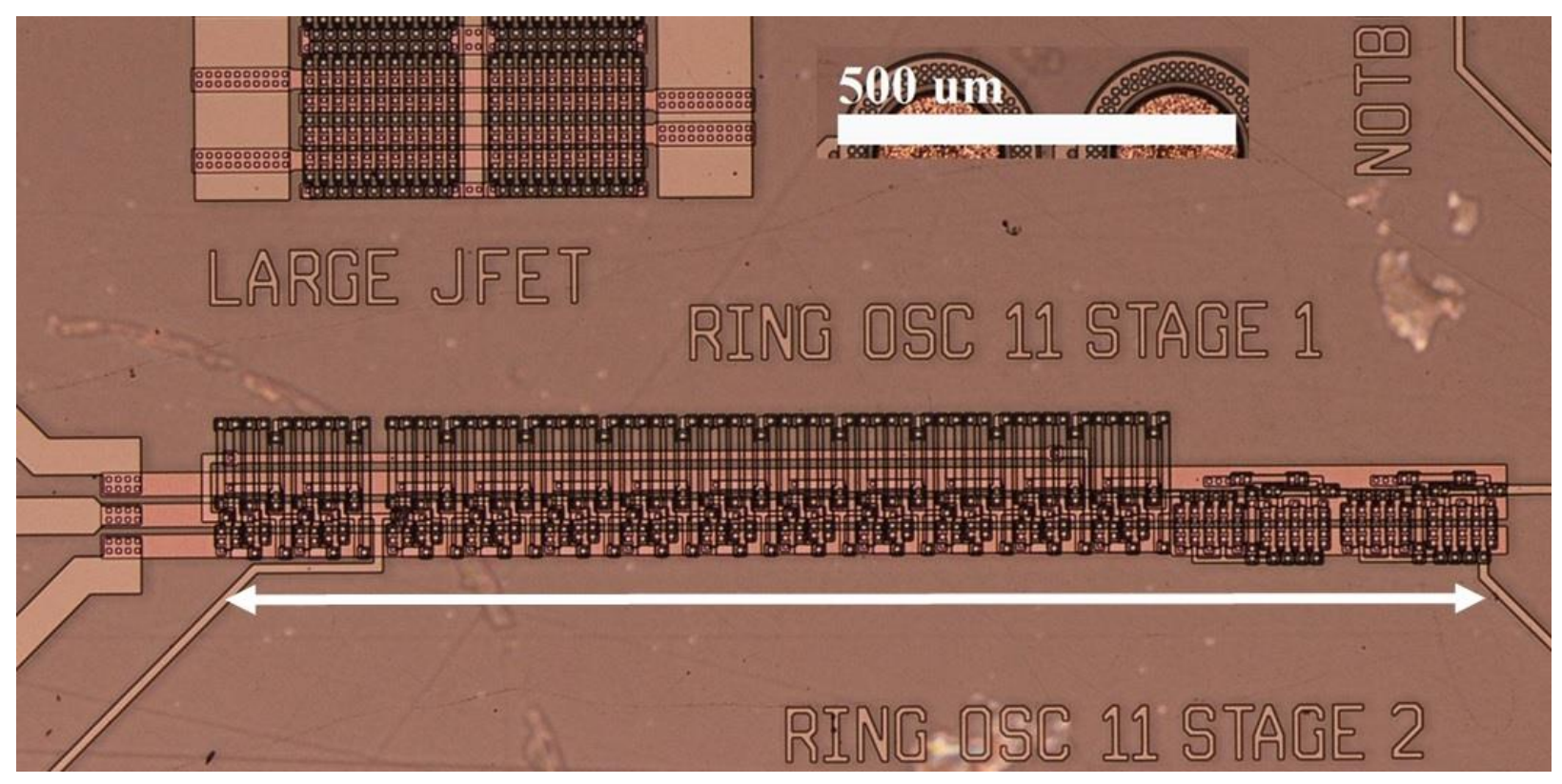




\section{Capacitive Pressure Sensor}

Capacitive pressure sensor developed by Sporian Microsystems

- Parallel plate capacitor design

- SICN membrane

- Superior oxidation/corrosion resistance at temperatures up to $1500^{\circ} \mathrm{C}$

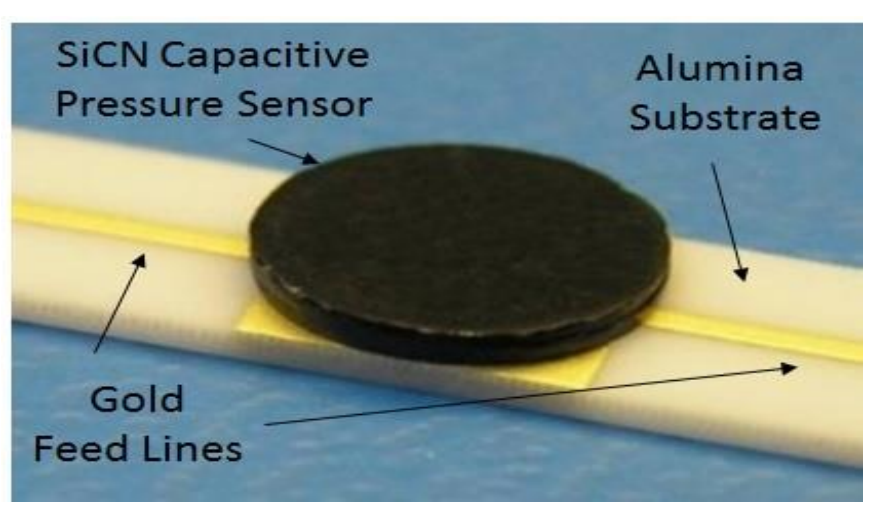

- One electrode of the capacitive sensor is fabricated on the deflecting membrane that forms a sealed cavity and the second electrode to the gold lead on the alumina substrate

- As pressure increases the capacitance of the sensor decreases

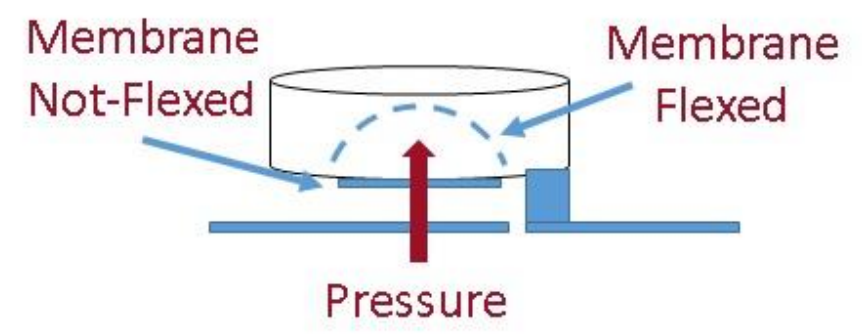




\section{Capacitive Pressure Sensor}

- Characterized from 0 to 300 psi at $25^{\circ} \mathrm{C}$ in steps of 50 psi with an Agilent 1500A Semiconductor Device Analyzer and customized pressure test fixture

- Custom built standards used to ensure accuracy

- At 0 psi the sensor has an initial capacitance of $3.76 \mathrm{pF}$ and reduces to $1.75 \mathrm{pF}$ at 300 psi

- Sensor sensitivity of 6.67 $\mathrm{fF} / \mathrm{psi}$ across pressure range

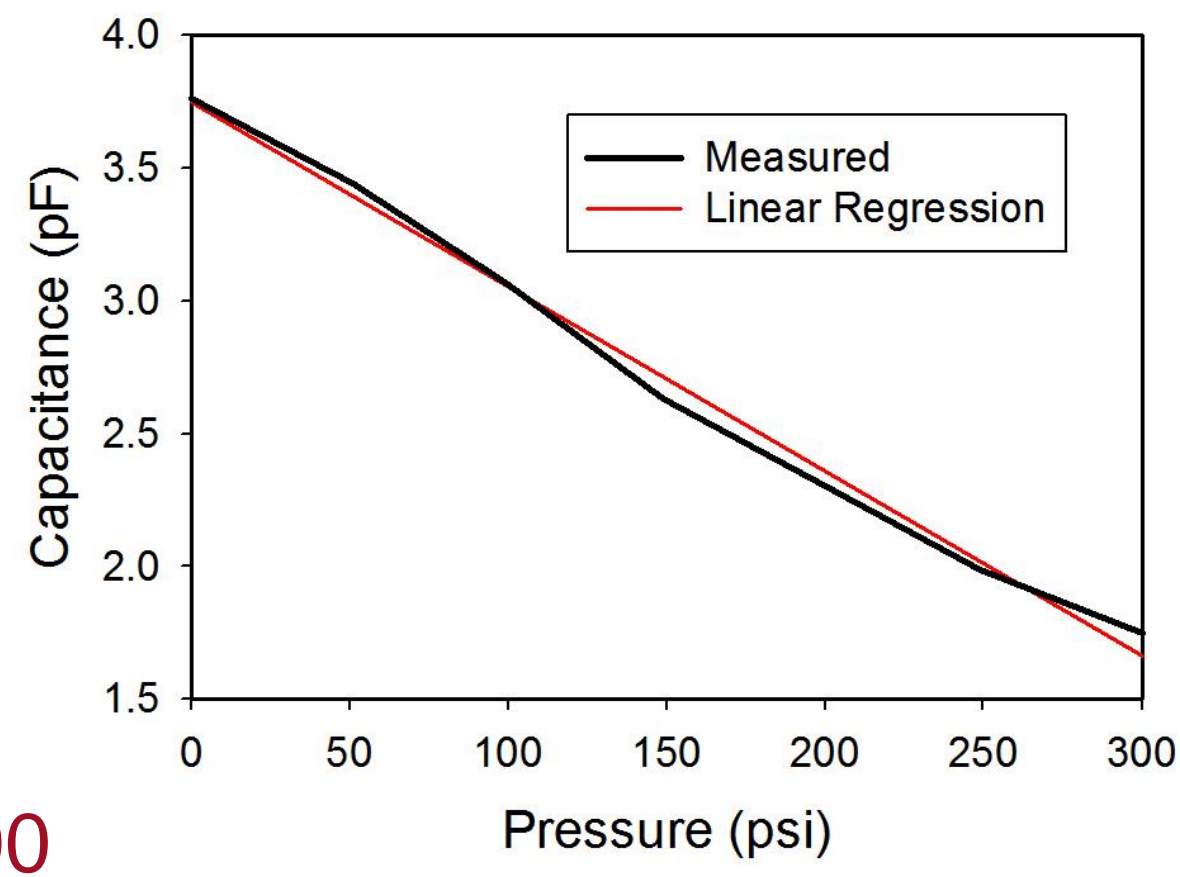

Sensor is extremely linear from 0 to 300 psi Correlation coefficient " $r$ " was 0.999 


\section{Pressure Sensor System}

The pressure sensor system consisted of two 11-stage ring oscillators and SiCN pressure sensor

- Osc1 has the sensor connected to a node in the feedback loop and then to ground

- Osc2 left unbounded

- Osc1 varies as a function of both pressure and temperature and Osc2 provides a pressureindependent reference frequency

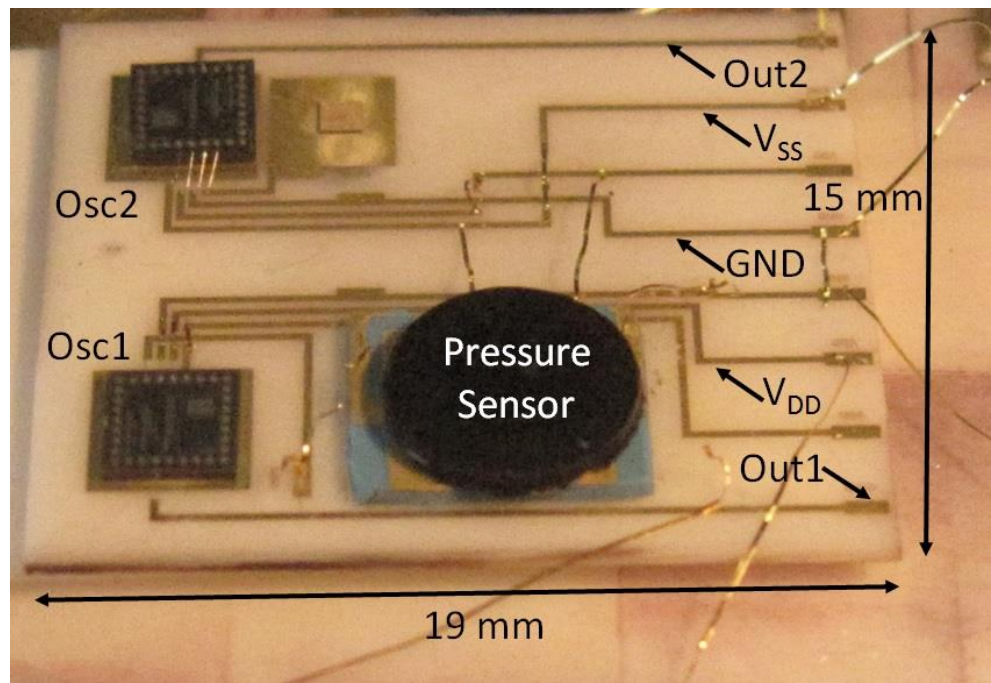
which can be used to temperature compensate Osc1 


\section{Pressure Sensor System}

\section{Pressure sensor system characterization}

- Two Agilent E4440A spectrum analyzers (where each analyzer is dedicated to one oscillator output)

- DC power supply

- Computer with LabVIEW program for data acquisition

- Custom built high temperature pressure test fixture 


\section{Ring Oscillator Reliability}

- Oscillators initially tested in an oven up to $500^{\circ} \mathrm{C}$ prior to connecting the pressure sensor to Osc1

- LabVIEW program recorded the $\mathrm{f}_{0}$ and corresponding magnitude for Osc1 and Osc2 as a function of temperature and time

- The temperature was ramped up $3^{\circ} \mathrm{C} / \mathrm{min}$ to $500^{\circ} \mathrm{C}$ were the temperature dwelled for 5 hours and then ramped back down $3^{\circ} \mathrm{C} / \mathrm{min}$ to room temperature

- During test $\mathrm{V}_{\mathrm{DD}}$ was set to $+25 \mathrm{~V}$ and $\mathrm{V}_{\mathrm{SS}}$ was set to $-25 \mathrm{~V}$, and both supply voltages remained constant throughout the test

- The test was repeated the following day to study the reliability and consistency of the devices 


\section{Ring Oscillator Reliability}

\begin{tabular}{|c|c|c|c|c|}
\hline & Day1 & Day1 & Day2 & Day2 \\
\hline & $25 \mathrm{C}$ & $500 \mathrm{C}$ & $25 \mathrm{C}$ & $500 \mathrm{C}$ \\
\hline Osc1 & 893.5 & 204.6 & 901.1 & 206.4 \\
& $\mathrm{kHz}$ & $\mathrm{kHz}$ & $\mathrm{kHz}$ & $\mathrm{kHz}$ \\
\hline Osc2 & 956.1 & 216.3 & 963.9 & 218.3 \\
& $\mathrm{kHz}$ & $\mathrm{kHz}$ & $\mathrm{kHz}$ & $\mathrm{kHz}$ \\
\hline
\end{tabular}

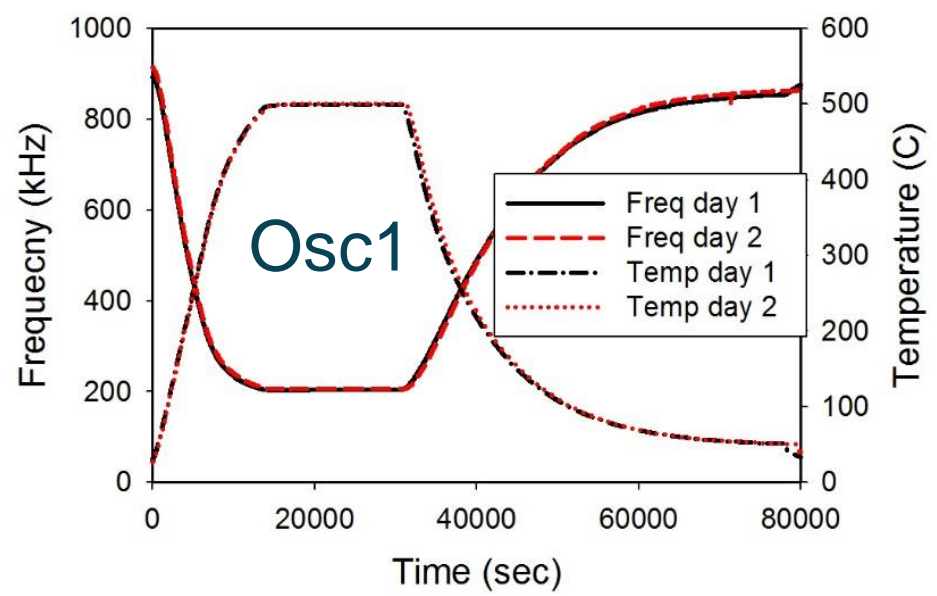

- Osc $1 \mathrm{f}_{0}$ changed 77.1 and $77.2 \%$ from 25 to $500^{\circ} \mathrm{C}$ on Day1 and Day2, respectively

- $\quad$ Osc $2 \mathrm{f}_{0}$ changed 77.4 and $77.3 \%$ from 25 to $500^{\circ} \mathrm{C}$ on Day 1 and Day2, respectively

Note: Even though Osc1 and Osc2 are identical in layout and fab, their electrical performance differs slightly due to location on wafer

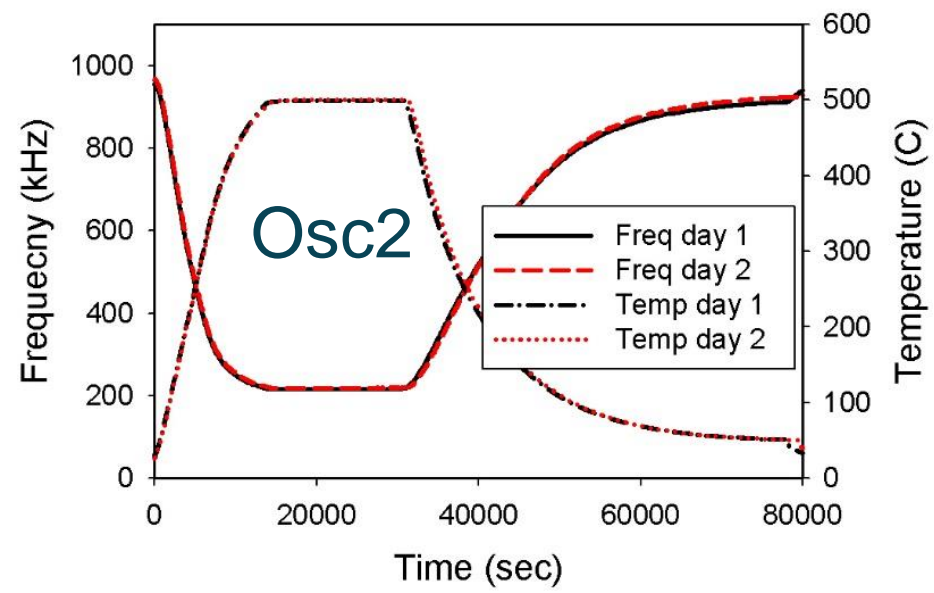




\section{Ring Oscillator Reliability}

\begin{tabular}{|c|c|c|c|c|}
\hline & Day1 & Day1 & Day2 & Day2 \\
\hline & $25 \mathrm{C}$ & $500 \mathrm{C}$ & $25 \mathrm{C}$ & $500 \mathrm{C}$ \\
\hline \multirow{2}{*}{ Osc1 } & -32.6 & -28.3 & -29.8 & -28.1 \\
& $\mathrm{dBm}$ & $\mathrm{dBm}$ & $\mathrm{dBm}$ & $\mathrm{dBm}$ \\
\hline \multirow{2}{*}{ Osc2 } & -27.8 & -28.4 & -27.7 & -29.3 \\
& $\mathrm{dBm}$ & $\mathrm{dBm}$ & $\mathrm{dBm}$ & $\mathrm{dBm}$ \\
\hline
\end{tabular}

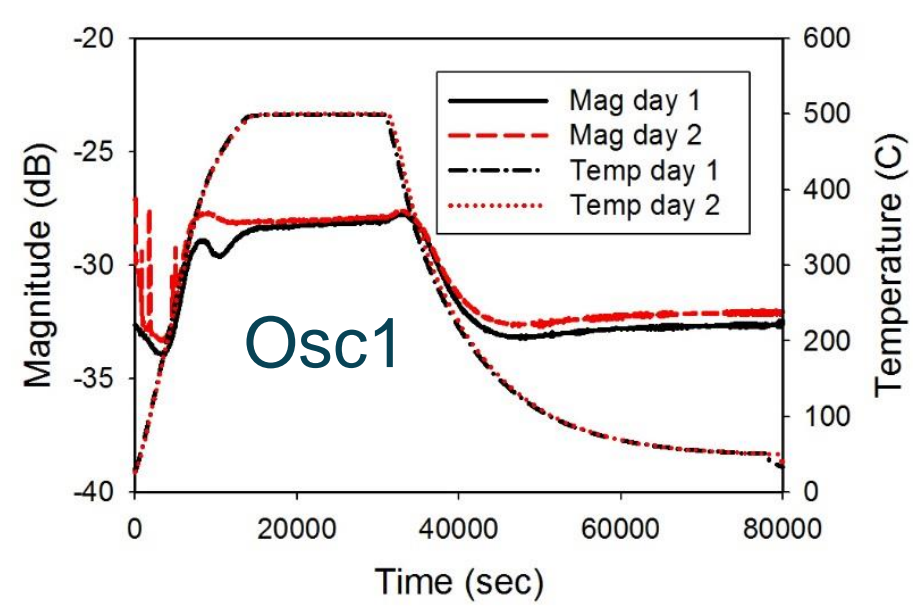

The initial decrease in amplitude is due to the increase output resistance as the temperature increases. However as the frequency decreases the amplitude increases and becomes the predominant factor.

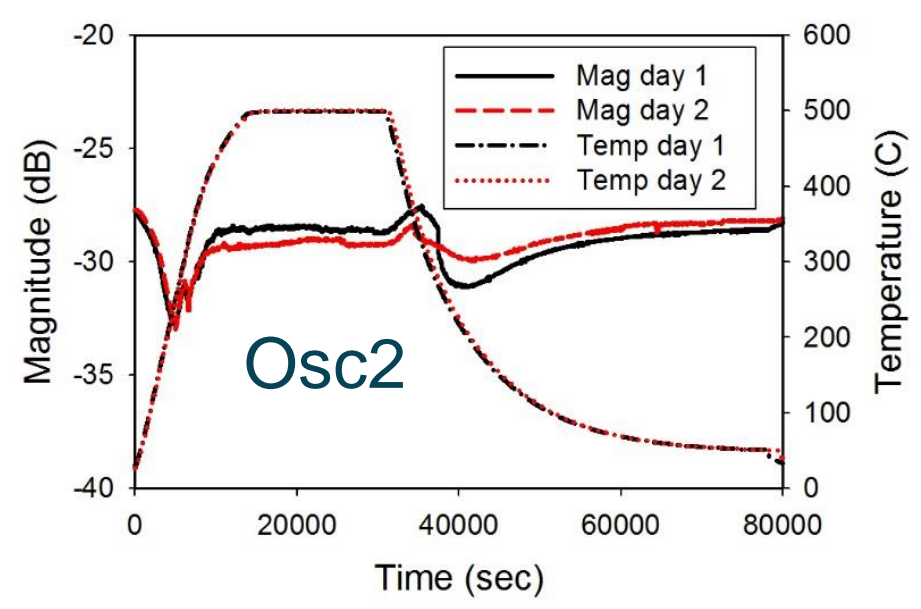




\section{Sensor System Characterization}

- Measured from 25 to $500^{\circ} \mathrm{C}$ from 0 to 300 psi in steps of 50 psi

- Custom built high temperature/pressure test fixture

- LabVIEW program recorded the $\mathrm{f}_{0}$ and corresponding magnitude for Osc1 and Osc2 as a function of temperature and

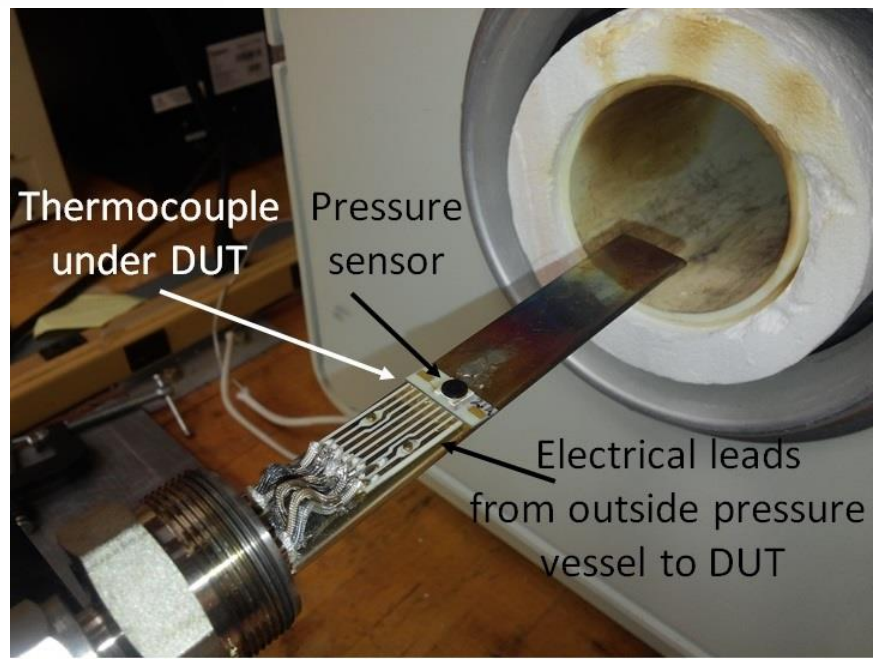
pressure

- During test $\mathrm{V}_{\mathrm{DD}}$ was set to $+25 \mathrm{~V}$ and $V_{S S}$ was set to $-25 \mathrm{~V}$, and both supply voltages remained constant throughout the test

- Thermocouple placed directly under DUT to facilitate accurate temperature reading

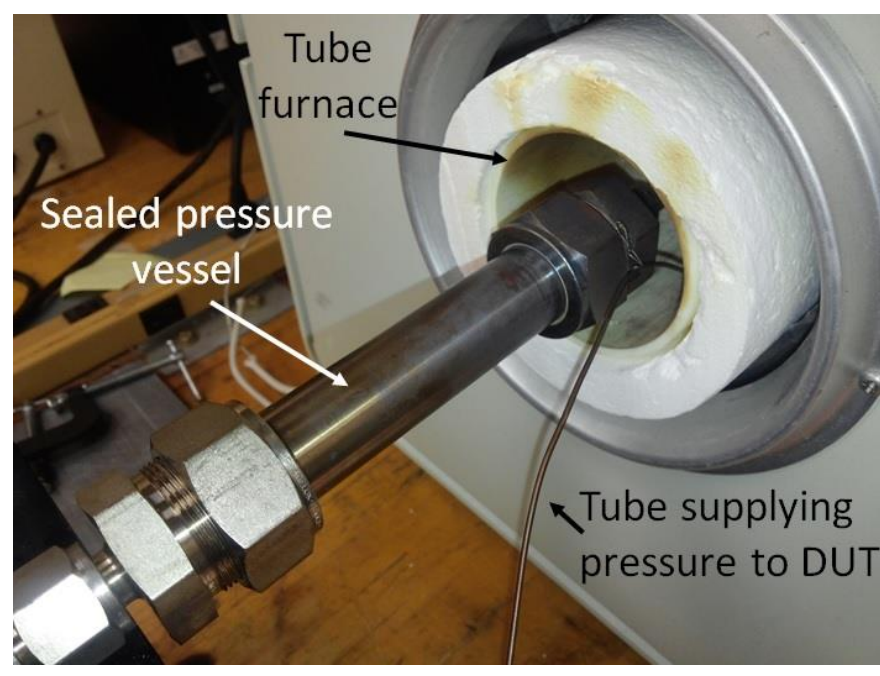




\section{Senor System Characterization}

The measured oscillation frequencies for Osc1 (with sensor for pressures of 0 and 300 psi and without sensor) and Osc2 from 25 to $500^{\circ} \mathrm{C}$

- Oscillation frequencies decrease with increasing $T$ due to decrease SiC JFET and resistor current conduction

- Osc1 without sensor falls just below Osc1 with sensor for $\mathrm{T}>350^{\circ} \mathrm{C}$, believed to be due to unaccounted parasitics from pressure sensor at high temperature

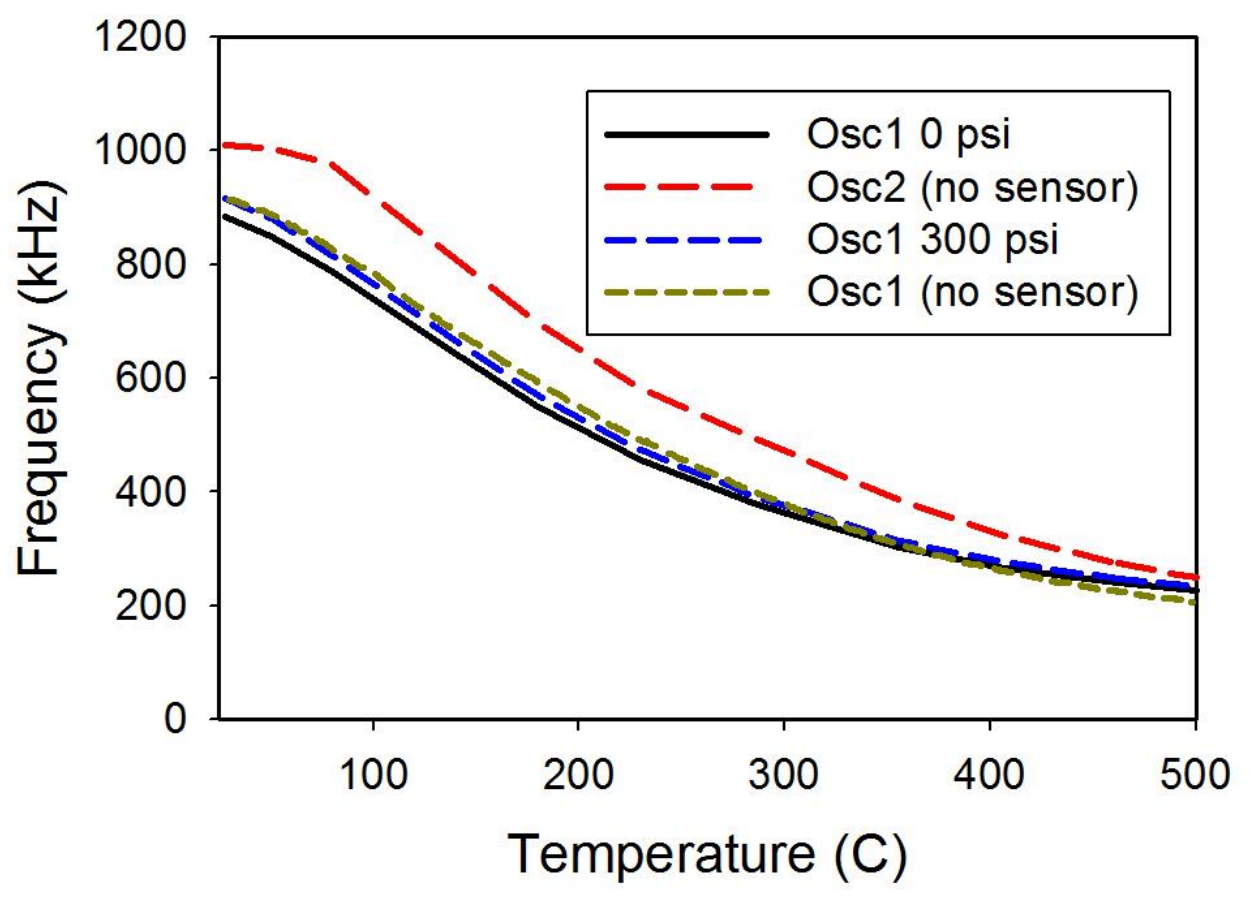




\section{Sensor System Characterization}

Measured response Osc1 as a function of temperature and pressure

- The $\Delta f_{0}\left(f_{300 p s i}-f_{\text {Opsi }}\right)$ decreases as the temperature increases

- The \% Change remains relatively the same with minor fluctuations which is due to the a non-optimal $\pm 2^{\circ} \mathrm{C}$ fluctuation in furnace temperature

- The Rate of Change decreases as temperature increases due to the decreasing $\Delta \mathrm{f}_{0}$ over the consistent pressure range of 0 to $300 \mathrm{psi}$

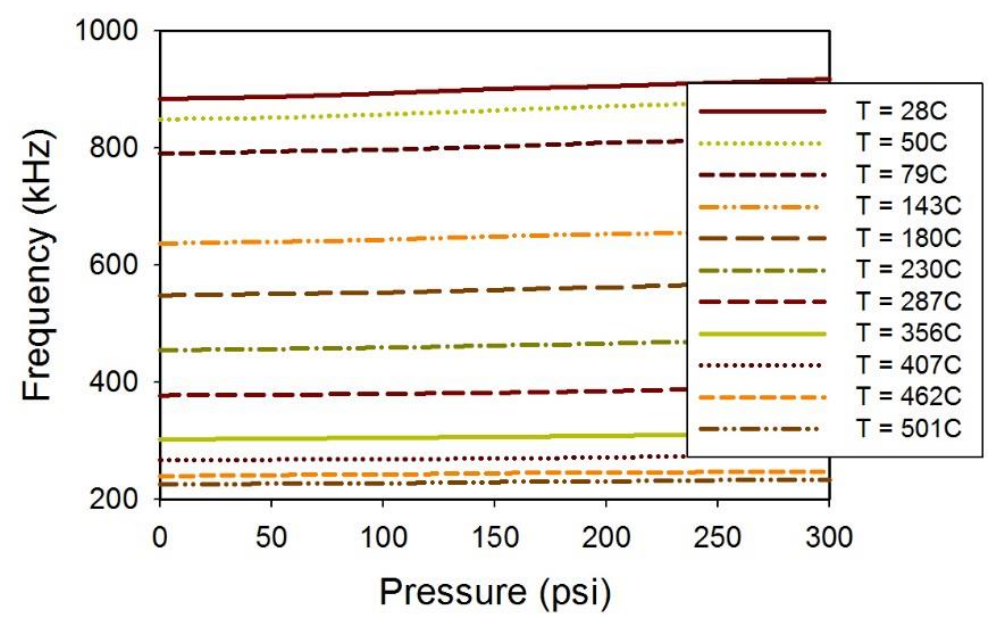

\begin{tabular}{|c|c|c|c|}
\hline $\begin{array}{c}\text { Temp } \\
\left({ }^{\circ} \mathrm{C}\right)\end{array}$ & $\begin{array}{c}\Delta \mathbf{f}_{0} \\
(\mathrm{kHz})\end{array}$ & $\begin{array}{c}\% \\
\text { Change } \\
\text { 0-300psi }\end{array}$ & $\begin{array}{c}\text { Rate of Change } \\
\text { kHz/psi }\end{array}$ \\
\hline 28 & 34 & 3.85 & 0.113 \\
\hline 50 & 32 & 3.78 & 0.107 \\
\hline 79 & 28 & 3.54 & 0.093 \\
\hline 143 & 21 & 3.30 & 0.070 \\
\hline 180 & 21 & 3.83 & 0.070 \\
\hline 230 & 18 & 3.96 & 0.060 \\
\hline 287 & 13 & 3.44 & 0.043 \\
\hline 356 & 11 & 3.63 & 0.037 \\
\hline 407 & 9 & 3.37 & 0.030 \\
\hline 462 & 8 & 3.33 & 0.027 \\
\hline 501 & 8 & 3.54 & 0.026 \\
\hline
\end{tabular}




\section{Sensor System Characterization}

\section{Measured response of the sensor with temperature compensation}

- The fundamental frequency, $\mathrm{f}_{0}$, of Osc2 minus Osc1 from 0 to 300 psi over the temperature range of 25 to $500^{\circ} \mathrm{C}$ decreases as pressure increases.

- Subtracting Osc1 from Osc2 effectively removes the temperature dependence with respect to pressure changes leaving only the pressure change as a function of frequency.

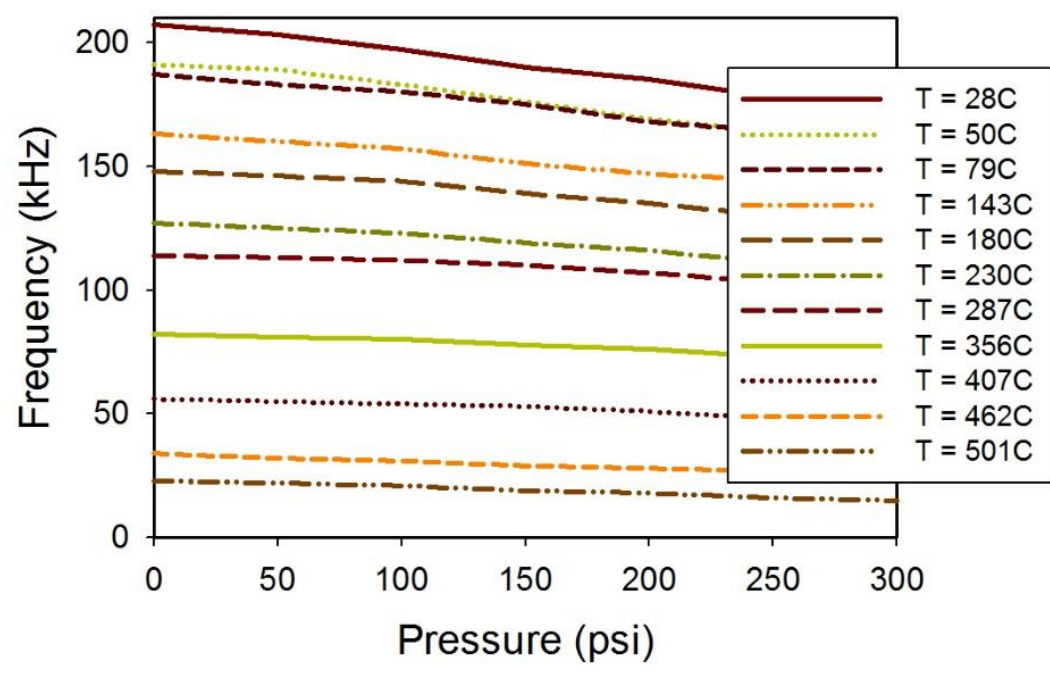

$f_{02}$ minus $f_{01 \text { (0 to 300pis) }}$ removes temperature dependency of the sensing system 


\section{Summary}

- Demonstrated a Novel pressure sensing system utilizing a SiCN capacitive pressure sensor and twin $\mathrm{SiC} I \mathrm{IC}$ ring oscillators over a temperature range of 25 to $500^{\circ} \mathrm{C}$ from 0 to 300 psi

- 1st order spice model were used to design the sensor with very good accuracy as a function of temperature

- Demonstrated the reliability and consistency of the ring oscillators

- The \% Change of the sensing system remained relatively the same with minor fluctuations which is due to the a non-optimal $\pm 2^{\circ} \mathrm{C}$ fluctuation in furnace temperature

- The Rate of Change of the sensing system decreased as temperature increased and is due to the decreasing $\Delta \mathrm{f}_{0}$ over the consistent pressure range of 0 to $300 \mathrm{psi}$

- The temperature dependency of the sensing system can effectively be removed by subtracting Osc2 $\left(\mathrm{f}_{0}\right)$ from Osc1 $\left(\mathrm{f}_{0}\right)$

- This is the initial design of a pressure sensing system that will detect the change in pressure on the surface of Venus 


\section{Acknowledgements}

NASA Glenn Research Center

Elizabeth Mcquaid, Nick Varaljay and Liang-Yu Chen

Long-Life In-Situ Solar System Explorer (LLISSE) and Transformative Tools and Technologies projects

\section{Sporian Microsystems}

Keven Harsh, Evan Pilant and Mike Usrey 


\section{Thank you}

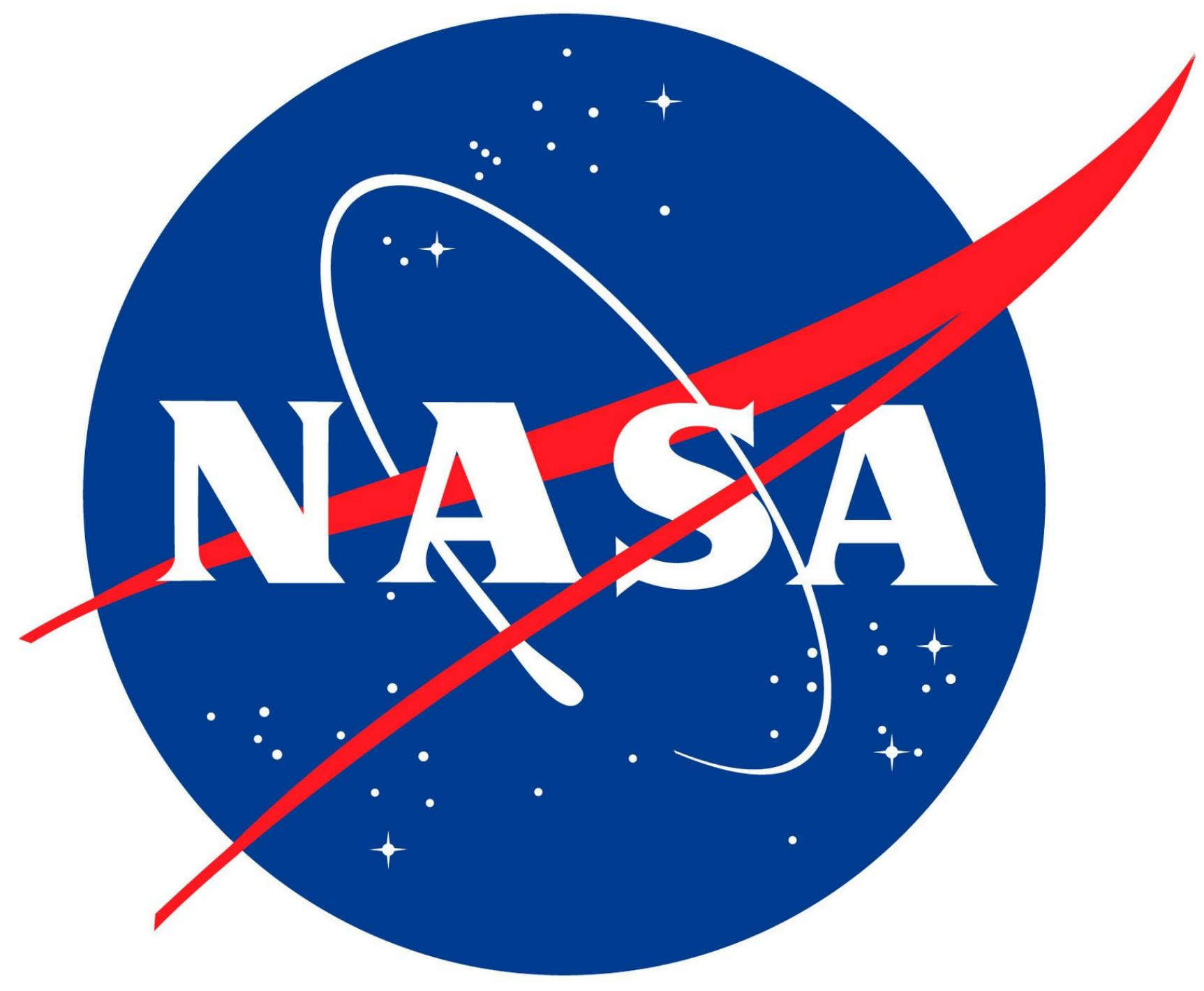

